\title{
A new intuitionistic fuzzy definiteness norm
}

\author{
Krassimir Atanassov ${ }^{1}$ and Peter Vassilev ${ }^{2}$ \\ ${ }^{1}$ Department of Bioinformatics and Mathematical Modelling, \\ Institute of Biophysics and Biomedical Engineering, \\ Bulgarian Academy of Sciences \\ Acad. G. Bonchev Str., Block 105, Sofia-1113, Bulgaria \\ e-mail: krat@bas.bg \\ ${ }^{2}$ Department of Bioinformatics and Mathematical Modelling, \\ Institute of Biophysics and Biomedical Engineering, \\ Bulgarian Academy of Sciences \\ Acad. G. Bonchev Str., Block 105, Sofia-1113, Bulgaria \\ e-mail: peter.vassilev@gmail.com
}

Received: 14 August 2020

Accepted: 27 September 2020

Abstract: In the present paper we introduce a new intuitionistic fuzzy norm over intuitionistic fuzzy pairs and study some of its properties.

Keywords: Intuitionistic fuzzy sets, Norms, Modal operators.

2010 Mathematics Subject Classification: 03E72.

\section{Introduction}

The Intuitionistic Fuzzy Pair (see [3,6]) is an object with the form $\langle a, b\rangle$, where $a, b \in[0,1]$ and $a+b \leq 1$, that is used as an evaluation of some object or process.

The most important geometrical interpretation of the intuitionistic fuzzy pairs is shown on Figure 1.

For the needs of the discussion below, following [1,3], we define the notion of Intuitionistic Fuzzy Tautological Pair by:

$x$ is an intuitionistic fuzzy tautological pair if and only if (iff) $a \geq b$, while $p$ is a Tautological Pair iff $a=1$ and $b=0$. 


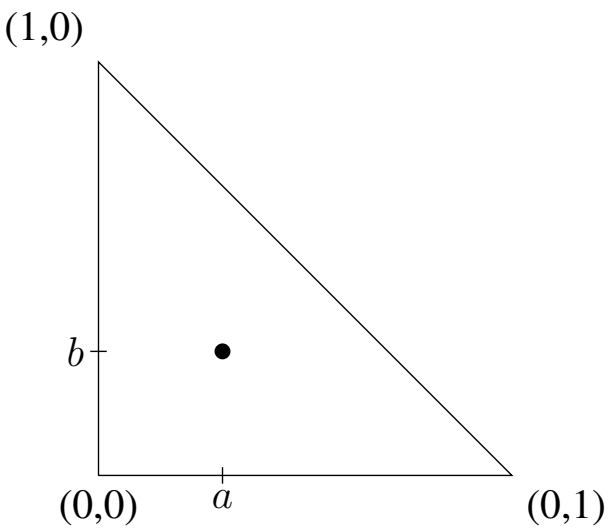

Figure 1. Representation of an intuitionistic fuzzy pair

Let us have two intuitionistic fuzzy pairs $x=\langle a, b\rangle$ and $y=\langle c, d\rangle$. Following [6], we define the relations

$$
\begin{array}{lll}
x<y & \text { iff } & a<c \text { and } b>d \\
x \leq y & \text { iff } & a \leq c \text { and } b \geq d \\
x>y & \text { iff } & a>c \text { and } b<d \\
x \geq y & \text { iff } & a \geq c \text { and } b \leq d \\
x=y & \text { iff } & a=c \text { and } b=d
\end{array}
$$

There are three types of operators over intuitionistic fuzzy pairs, introduced in [6]. The first of them is of modal type and we will introduced only them.

Let as above, $x=\langle a, b\rangle$ be an intuitionistic fuzzy pair and let $\alpha, \beta \in[0,1]$. Then the modal type of operators defined over $x$ have the forms:

$$
\begin{aligned}
& \square x=\langle a, 1-a\rangle \\
& \nabla x=\langle 1-b, b\rangle \\
& D_{\alpha}(x)=\langle a+\alpha \cdot(1-a-b), b+(1-\alpha) \cdot(1-a-b)\rangle \\
& F_{\alpha, 1-\alpha}(x)=\langle a+\alpha \cdot(1-a-b), b+\beta \cdot(1-a-b)\rangle, \text { where } \alpha+\beta \leq 1 \\
& G_{\alpha, \beta}(x)=\langle\alpha \cdot a, \beta \cdot b\rangle \\
& H_{\alpha, \beta}(x)=\langle\alpha \cdot a, b+\beta \cdot(1-a-b)\rangle \\
& H_{\alpha, \beta}^{*}(x)=\langle\alpha \cdot a, b+\beta \cdot(1-\alpha \cdot a-b)\rangle \\
& J_{\alpha, \beta}(x)=\langle a+\alpha \cdot(1-a-b), \beta \cdot b\rangle \\
& J_{\alpha, \beta}^{*}(x)=\langle a+\alpha \cdot(1-a-\beta . b), \beta \cdot b\rangle
\end{aligned}
$$




\section{Main results}

Let the intuitionistic fuzzy pair $x=\langle a, b\rangle$ be given. For it, we define

$$
\delta(x) \equiv\left\langle L_{\delta}(x), R_{\delta}(x)\right\rangle=\langle(2-a-2 b) a,(2-2 a-b) b\rangle .
$$

Theorem 1. The definition of $\delta$ is correct.

Proof. By assumption $x=\langle a, b\rangle$ is an intuitionistic fuzzy pair. We will prove that $y=\langle(c+1-$ $a-b) a,(d+1-a-b) b\rangle$ is also an intuitionistic fuzzy pair for all $c, d \in[0,1]$, and hence also for the particular choice $c=1-b$ and $d=1-a$. First, we observe that since $x$ is an intuitionistic fuzzy pair $1-a-b \geq 0$, and from $c \geq 0$ and $d \geq 0$, we have that

$$
0 \leq \min ((c+1-a-b) a,(d+1-a-b) b) \leq \max ((c+1-a-b) a,(d+1-a-b) b) .
$$

Since we have

$$
\max ((c+1-a-b) a,(d+1-a-b) b) \leq(c+1-a-b) a+(d+1-a-b) b
$$

to show the correctness we need to establish that

$$
(c+1-a-b) a+(d+1-a-b) b \leq 1 .
$$

After rewriting we get

$$
(a+b)(1-a-b)+\underbrace{a c+b d}_{\leq a+b} \leq(a+b)(1-a-b)+a+b \leq 1
$$

The last is equivalent to

$$
0 \leq 1-a-b-(1-a-b)(a+b)=(1-a-b)^{2},
$$

hence it is always true

Therefore, the definition of $\delta$ is correct.

The geometrical interpretation of $\delta(x)$ is given on Figure 2 .

Using (1), we check directly the following equalities:

$$
\begin{gathered}
\delta(\langle 0,1\rangle)=\langle 0,1\rangle, \\
\delta(\langle 0,0\rangle)=\langle 0,0\rangle, \\
\delta\left(\left\langle\frac{1}{2}, \frac{1}{2}\right\rangle\right)=\left\langle\frac{1}{4}, \frac{1}{4}\right\rangle, \\
\delta(\langle 1,0\rangle)=\langle 1,0\rangle, \\
\delta(\langle 0, b\rangle)=\langle 0,(2-b) b\rangle, \\
\delta(\langle a, 0\rangle)=\langle(2-a) a, 0\rangle, \\
\delta(\langle a, a\rangle)=\langle(2-3 a) a,(2-3 a) a\rangle,
\end{gathered}
$$

for each $a \in[0,1]$. 


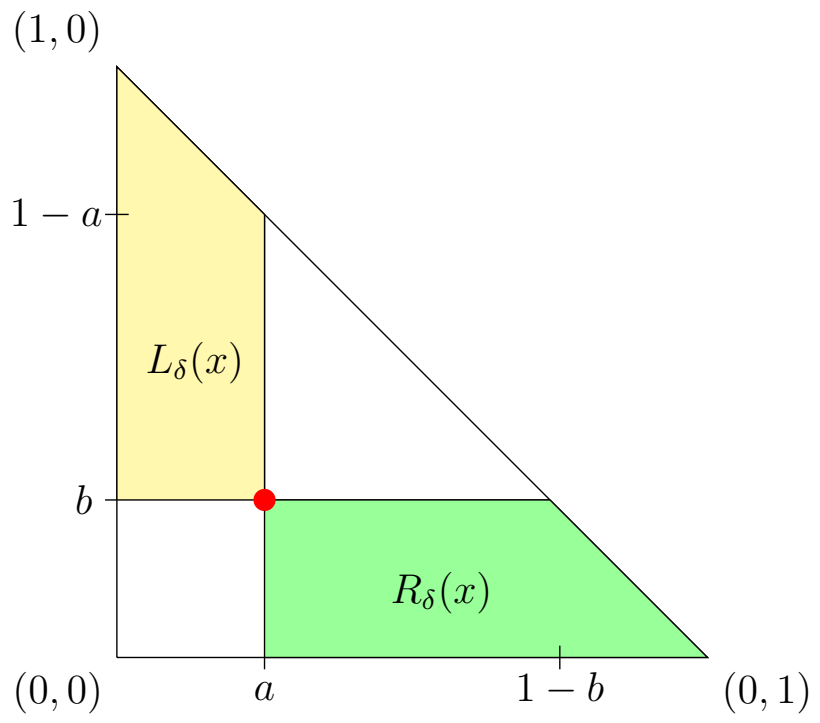

Figure 2. The geometrical interpretation of $\delta(x)$

Theorem 2. For each intuitionistic fuzzy pair $x$, it is an intuitionistic fuzzy tautological pair if and only if (iff) $\delta(x)$ is an intuitionistic fuzzy tautological pair; $x$ is a tautological pair iff $\delta(x)$ is a tautological pair.

Proof. Let $x$ be an IFT. We see that

$$
(2-a-2 b) a-(2-2 a-b) b=(a-b)(1-a+1-b)
$$

Since $2-a-b=1+1-a-b>0$, the validity of the Theorem follows .

By analogy with the above definitions of intuitionistic fuzzy tautological pair and tautological pair, for a first time we define the notion of Intuitionistic Fuzzy False Pair by:

$$
x \text { is an intuitionistic fuzzy false pair iff } a<b,
$$

while $x$ is a False Pair iff $a=0$ and $b=1$. Now, we can prove similarly to Theorem 2 the following two assertions

Theorem 3. For each intuitionistic fuzzy pair $x$, it is an intuitionistic fuzzy false pair iff $\delta(x)$ is an intuitionistic fuzzy false pair; $x$ is a FP iff $\delta(x)$ is a FP.

Theorem 4. For intuitionistic fuzzy pair intuitionistic fuzzy pair $x, \delta(x)=x$ iff $x=\langle 0,1\rangle,\langle 0,0\rangle$, $\langle 1,0\rangle,\left\langle\frac{1}{3}, \frac{1}{3}\right\rangle$.

Theorem 5. For each intuitionistic fuzzy pair $x$

$$
\delta(\neg x)=\neg \delta(x) .
$$

Proof. We check sequentially:

$$
\begin{aligned}
\delta(\neg x) & =\delta(\neg\langle a, b\rangle) \\
& =\delta(\langle b, a\rangle)=\langle(2-2 a-b) b,(2-2 b-a) a\rangle \\
& =\neg\langle(2-a-2 b) a,(2-2 a-b) b\rangle=\neg \delta(x) .
\end{aligned}
$$


Therefore,

$$
\neg \delta(\neg x)=\delta(x) .
$$

Theorem 6. For each intuitionistic fuzzy pair $x$

$$
\begin{gathered}
\delta(\square x)=\left\langle a^{2},(1-a)^{2}\right\rangle, \\
\delta(\diamond x)=\left\langle(1-b)^{2}, b^{2}\right\rangle .
\end{gathered}
$$

Proof. Let $x$ be an intuitionistic fuzzy pair. Then

$$
\begin{aligned}
\delta(\square x) & =\delta(\langle a, 1-a\rangle) \\
& =\langle(2-a-2+2 a) a,(2-2 a-1+a)(1-a)\rangle \\
& =\left\langle a^{2},(1-a)^{2}\right\rangle .
\end{aligned}
$$

The second equality is checked in the same manner.

Let us define for each intuitionistic fuzzy pair $\langle a, b\rangle$

$$
p=1-a-b .
$$

Theorem 7. For each intuitionistic fuzzy pair $x$ and for $\alpha \in[0,1]$ :

$$
\delta\left(D_{\alpha} x\right)=\left\langle(a+\alpha p)^{2},(b+(1-\alpha) p)^{2}\right\rangle .
$$

Proof. Let $x$ be an intuitionistic fuzzy pair. Then

$$
\begin{aligned}
& \delta\left(D_{\alpha} x\right)=\delta(\langle a+\alpha p, b+(1-\alpha) p\rangle) \\
& =\langle(2-a+\alpha p-2 b-2(1-\alpha) p)(a+\alpha p),(2-b+(1-\alpha) p-2 a-2 \alpha p)(b+(1-\alpha) p)\rangle \\
& =\langle(2-a-2 b-(2-\alpha) p)(a+\alpha p),(2-2 a-b-(1-\alpha) p)(b+(1-\alpha) p)\rangle \\
& =\left\langle(a+\alpha p)^{2},(b+(1-\alpha) p)^{2}\right\rangle .
\end{aligned}
$$

When $\alpha=0$, then (6) is transformed to (2), because of (5), while when $\alpha=1$, then (6) is transformed to (3).

Theorem 8. For each intuitionistic fuzzy pair $x$ and for $\alpha, \beta \in[0,1]$, such that $\alpha+\beta \leq 1$ :

$$
\delta\left(F_{\alpha, \beta}(x)\right)=\langle(a+(2-\alpha-2 \beta) p)(a+\alpha p),(b+(2-2 \alpha-\beta) p)(b+\beta p)\rangle .
$$

Proof. Let $x$ be an intuitionistic fuzzy pair. Then

$$
\begin{gathered}
\delta\left(F_{\alpha, \beta} x\right)=\delta(\langle a+\alpha p, b+\beta p\rangle) \\
=\langle(2-a-\alpha p-2 b-2 \beta p)(a+\alpha p),(2-2 a-2 \alpha p-b-\beta p)(b+\beta p)\rangle \\
=\langle(2-2 a-2 b-2 p+a+2 p-\alpha p-2 \beta p)(a+\alpha p),(2-2 a-2 b-2 p+b+2 p-2 \alpha p-\beta p)(b+\beta p)\rangle \\
=\langle(a+2 p-\alpha p-2 \beta p)(a+\alpha p),(b+2 p-2 \alpha p-\beta p)(b+\beta p)\rangle \\
=\langle(a+(2-\alpha-2 \beta) p)(a+\alpha p),(b+(2-2 \alpha-\beta) p)(b+\beta p)\rangle .
\end{gathered}
$$


When we put $\beta=1-\alpha$, (7) is transformed to (6).

Let us for brevity assume below that $x \neq\langle 0, b\rangle,\langle a, 0\rangle$ for every $a, b \in[0,1]$.

Theorem 9. For an intuitionistic fuzzy pair $x$ such that $p>0,2 a+b \leq 1$ and $a+2 b \geq 1$, there exist $\alpha, \beta \in[0,1]$, for which

$$
H_{\alpha, \beta}(x)=\delta(x) .
$$

Proof. We must find $\alpha$ and $\beta$ such that:

$$
\begin{gathered}
(2-a-2 b) a=\alpha a, \\
(2-2 a-b) b=b+\beta(1-a-b) .
\end{gathered}
$$

Therefore,

$$
\alpha=2-a-2 b
$$

that by condition $a \neq 0$ and

$$
\alpha=2-a-2 b \leq 1,
$$

i.e., $\alpha \in[0,1]$, and from $p=1-a-b>0$ :

$$
\beta=\frac{(2-2 a-b) b-b}{1-a-b}=\frac{(1-2 a-b) b}{1-a-b}
$$

that also is in interval $[0,1]$.

We must mention that both conditions $2 a+b \leq 1$ and $a+2 b \geq 1$ imply inequality $a \leq b$.

Now, we see that

$$
\begin{gathered}
H_{2-a-2 b, \frac{(1-2 a-b) b}{1-a-b}}(x)=\left\langle(2-a-2 b) a, b+\frac{(1-2 a-b) b}{1-a-b}(1-a-b)\right\rangle \\
=\langle(2-a-2 b) a, b+(1-2 a-b) b\rangle=\langle(2-a-2 b) a,(2-2 a-b) b\rangle=\delta(x) .
\end{gathered}
$$

The area of the intuitionistic fuzzy pairs over which the operator $H_{\alpha, \beta}$ can be applied is shown on Figure 3.

Theorem 10. For an intuitionistic fuzzy pair $x$ such that $2 a+b \leq 1$ and $a+2 b \geq 1$, there are $\alpha, \beta \in[0,1]$, for which

$$
H_{\alpha, \beta}^{*}(x)=\delta(x) .
$$

The proof is similar to this of equality (8). Here,

$$
\begin{gathered}
\alpha=2-a-2 b, \\
\beta=\frac{(1-2 a-b) b}{(1-a-b)(1-a)+a b} .
\end{gathered}
$$

Obviously, for $a, b \neq 0,(1-a-b)(1-a)+a b>0$.

The area of the intuitionistic fuzzy pairs over which the operator $H_{\alpha, \beta}^{*}$ can be applied is shown on Figure 3. 


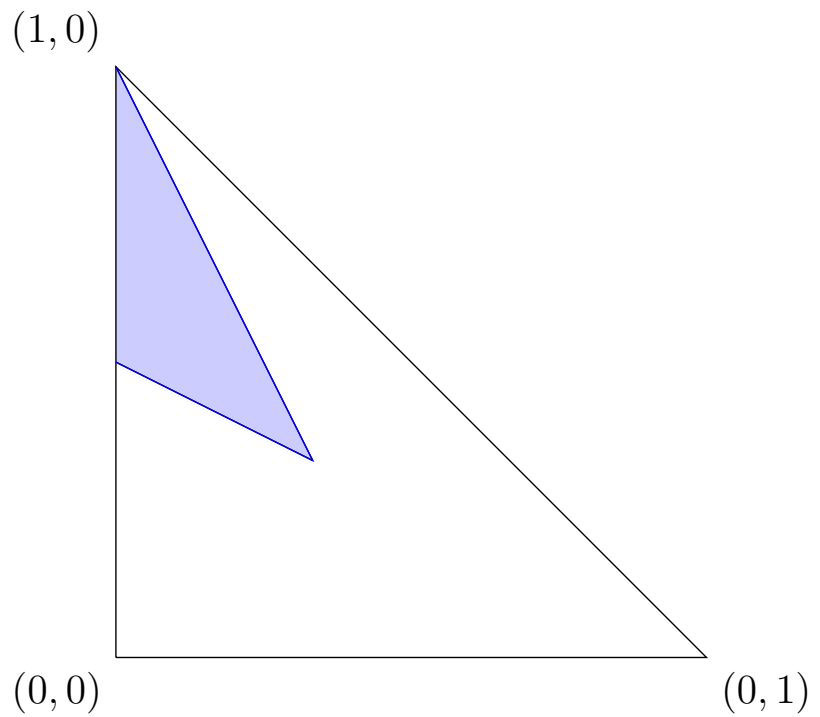

Figure 3. The area of application of operators $H_{\alpha, \beta}$ and $H_{\alpha, \beta}^{*}$ for intuitionistic fuzzy pairs

Theorem 11. For an intuitionistic fuzzy pair $x$ such that $p>0, a+2 b \leq 1$ and $2 a+b \geq 1$, there are $\alpha, \beta \in[0,1]$, for which

$$
J_{\alpha, \beta}(x)=\delta(x) .
$$

The proof is similar to this of equality (8). Here,

$$
\begin{gathered}
\alpha=\frac{(1-a-2 b) a}{1-a-b}, \\
\beta=2-2 a-b,
\end{gathered}
$$

and both conditions $a+2 b \leq 1$ and $2 a+b \geq 1$ imply inequality $a \geq b$.

The area of the intuitionistic fuzzy pairs over which the operator $J_{\alpha, \beta}$ can be applied is shown on Figure 4.

Theorem 12. For an intuitionistic fuzzy pair $x$ such that $a+2 b \leq 1$ and $2 a+b \geq 1$, there are $\alpha, \beta \in[0,1]$, for which

$$
J_{\alpha, \beta}^{*}(x)=\delta(x)
$$

The proof is again similar to this of equality (8). Here,

$$
\begin{gathered}
\alpha=\frac{(1-a-2 b) a}{(1-a-b)(1-b)+a b}, \\
\beta=2-2 a-b .
\end{gathered}
$$

Again, for $a, b \neq 0,(1-a-b)(1-b)+a b>0$.

The area of the intuitionistic fuzzy pairs over which the operator $J_{\alpha, \beta}^{*}$ can be applied is shown on Figure 4. 
$(1,0)$

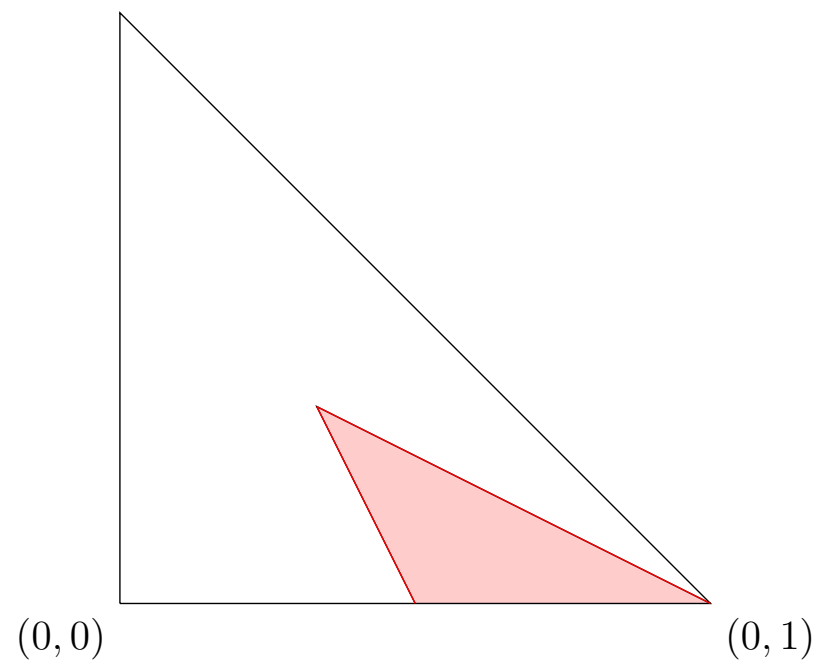

Figure 4. The area of application of operators $J_{\alpha, \beta}$ and $J_{\alpha, \beta}^{*}$ for intuitionistic fuzzy pairs

\section{Conclusion}

In future, new properties of the norm $\delta$ will be studied. It will be interesting, if it can be used for determining of the areas of positive and negative consonances in the intercriteria analysis (see $[2,4,5])$.

In this regard, the interested reader can see certain similarities between the newly proposed intuitionistic fuzzy norm and the approach adopted in [7] with the modified level operator $N_{\gamma}$. Graphically, that operator cuts off a smaller triangle from the overall intuitionistic fuzzy triangle, by producing a subset of an intuitionistic fuzzy set, which elements satisfy the condition for the ratio between their degrees of membership and their degrees of non-membership, respectively, to be greater or equal to a predefined number $\gamma>0$, i.e., the elements who membership is at least $\gamma$ times greater than their non-membership.

\section{Acknowledgements}

This research has been supported by the Bulgarian National Science Fund under Grant Ref. No. KP-06-N22/1/2018 “Theoretical research and applications of InterCriteria Analysis”.

\section{References}

[1] Atanassov, K. (1988). Two variants of intuitionistic fuzzy propositional calculus. Preprint IM-MFAIS-5-88, Sofia, 1988. Reprinted: Int. J. Bioautomation, 2016, 20(S1), S17-S26. 
[2] Atanassov, K. (2014). Index Matrices: Towards an Augmented Matrix Calculus, Springer, Cham.

[3] Atanassov, K. (2017). Intuitionistic Fuzzy Logics, Springer, Cham.

[4] Atanassov, K., Atanassova, V., \& Gluhchev, G. (2015). InterCriteria Analysis: Ideas and problems. Notes on Intuitionistic Fuzzy Sets, 21 (1), 81-88.

[5] Atanassov, K., Mavrov, D., \& Atanassova, V. (2014). Intercriteria Decision Making: A New Approach for Multicriteria Decision Making, Based on Index Matrices and Intuitionistic Fuzzy Sets. Issues in Intuitionistic Fuzzy Sets and Generalized Nets, 11, 1-8

[6] Atanassov, K., Szmidt, E., \& Kacprzyk, J. (2013). On Intuitionistic Fuzzy Pairs, Notes on Intuitionistic Fuzzy Sets, 19 (3), 1-13.

[7] Atanassova, V. (2017). New modified level operator $N_{\gamma}$ over intuitionistic fuzzy sets. Proc. of 12th International Conference on Flexible Query Answering Systems (FQAS 2017), London, UK, June 21-22, 2017, (Christiansen, H., Jaudoin, H., Chountas, P., Andreasen, T., \& Larsen, H. L. (Eds.), LNAI 10333, Springer, 209-214. 